\title{
Nonstoichiometric Low-Temperature Grown GaAs Nanowires
}

\author{
Adrian Díaz Álvarez, ${ }^{\dagger}$ Tao Xu, ${ }^{\dagger, \ddagger}$ Gözde Tütüncüoglu, ${ }^{\S}$ Thomas Demonchaux, ${ }^{\dagger}$ Jean-Philippe Nys, \\ Maxime Berthe, ${ }^{\dagger}$ Federico Matteini, ${ }^{\S}$ Heidi A. Potts, ${ }^{\S}$ David Troadec, ${ }^{\dagger}$ Gilles Patriarche, ${ }^{\|}$ \\ Jean-François Lampin, ${ }^{\dagger}$ Christophe Coinon, ${ }^{\dagger}$ Anna Fontcuberta i Morral, ${ }^{\S}$ Rafal E. Dunin-Borkowski, ${ }^{\perp}$ \\ Philipp Ebert, ${ }^{\perp}$ and Bruno Grandidier* ${ }^{\dagger}$

\footnotetext{
†Institut d’Electronique, de Microélectronique et de Nanotechnologies (IEMN), CNRS, UMR 8520, Département ISEN, 41 bd Vauban, 59046 Lille Cedex, France

¥Sino-European School of Technology, Shanghai University, 99 Shangda Road, Shanghai, 200444, People’s Republic of China

${ }^{\S}$ Laboratoire des Matériaux Semiconducteurs, Institut des Matériaux, Ecole Polytechnique Fédérale de Lausanne, CH-1015 Lausanne, Switzerland

"CNRS-Laboratoire de Photonique et de Nanostructures (LPN), Route de Nozay, 91460 Marcoussis, France

${ }^{\perp}$ Peter Grünberg Institut, Forschungszentrum Jülich GmbH, 52425 Jülich, Germany
}

\section{Supporting Information}

\begin{abstract}
The structural and electronic properties of nonstoichiometric low-temperature grown GaAs nanowire shells have been investigated with scanning tunneling microscopy and spectroscopy, pump-probe reflectivity, and cathodoluminescence measurements. The growth of nonstoichiometric GaAs shells is achieved through the formation of As antisite defects, and to a lower extent, after annealing, As precipitates. Because of the high density of atomic steps on the nanowire sidewalls, the Fermi level is pinned midgap, causing the ionization of the subsurface antisites and the formation of depleted regions around the As precipitates. Controlling their incorporation offers a way to obtain unique electronic and optical properties that depart from the ones found in conventional GaAs nanowires.
\end{abstract}

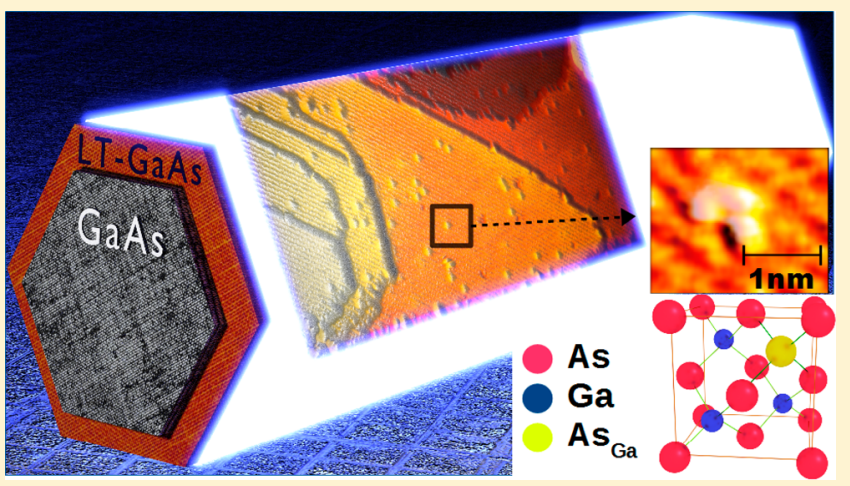

KEYWORDS: Nonstoichiometric GaAs, low-temperature growth, As antisite, As precipitates, nanowires, scanning tunneling microscopy

$\mathrm{T}$ he prospect of using semiconductor nanowires (NWs) as building blocks of miniaturized devices has triggered a continuous improvement of the material crystal quality and reproducibility. For instance, gaining control over the phase purity in III-V semiconductor nanowires ${ }^{1}$ has led to narrow photoluminescence emission in $\mathrm{GaAs} / \mathrm{GaAsSb}$ heterostructure nanowires $^{2}$ and to ballistic transport of electrons in InAs NW transistors. ${ }^{3}$ Similarly, the synthesis of defect-free interfaces in core-shell nanowires was a key achievement in getting higher collection efficiency of the photoexcited charge carriers in solar cells. ${ }^{4}$ While defects in semiconductor NWs are generally seen as deleterious, certain applications, however, rely on the intentional incorporation of point defects during growth. A prototypical semiconductor material, where defects matter for resistivity, sub-bandgap optical absorption, and carrier lifetime engineering, is GaAs grown at low temperature. Such a material is crucial for the development of photoconductive devices and, in particular, compact terahertz $(\mathrm{THz})$ sources and detectors with numerous applications in astronomy, communications, sensing, imaging, and medical diagnostics. ${ }^{5-8}$
The growth of GaAs NWs is usually based on the vaporliquid-solid (VLS) mechanism that involves the continuous supply of precursors into a seed particle maintained above a critical temperature. Whatever the method that is used ( $\mathrm{Au}$ assisted or Ga-assisted VLS mechanism), ${ }^{9,10}$ the eutectic temperature is higher than the threshold temperature at which nonstochiometric GaAs material can be grown and precludes the direct growth of high-quality nonstoichiometric LT-GaAs NWs. However, there is a great interest to build arrays of LT-GaAs NWs, since nanowires exhibit a significant enhancement of the $\mathrm{THz}$ emission with respect to thin films, without the need to insert them into bias photoconductive antenna structures. ${ }^{11-15}$ This may help to overcome the limitation of relatively low $\mathrm{THz}$ power encountered in thin LTGaAs layers. ${ }^{16,17}$

Here, we adopt the following core-shell strategy to synthesize a nonstoichiometric LT-GaAs NW: First GaAs

Received: May 7, 2015

Revised: July 30, 2015 
NWs of high quality are grown on Si substrates using the selfassisted VLS mechanism with molecular beam epitaxy. ${ }^{18}$ Then the temperature is reduced to grow a nonstoichiometric LTGaAs shell on the nanowire sidewalls. ${ }^{19}$ Here we examine the atomic scale structural and electronic properties of such LTGaAs NWs. Scanning tunneling microscopy (STM) of the NW sidewalls reveals the existence of point defects. Based on the structural and spectral signature of the defects, they are identified as isolated subsurface arsenic antisites, which are found to affect the optical properties of the NWs. In addition, nanoscale clusters surrounded by narrow space charge layers occur, consistent with the formation of metallic As precipitates in the semiconductor shell. Both features demonstrate the successful growth of nonstoichiometric GaAs NWs with excess arsenic. The here-demonstrated controlled incorporation of arsenic antisites and clusters provides the basis to combine ultrashort carrier lifetimes, high dark resistivity, and large nonlinear optical effects in NWs.

The synthesis of the NWs was achieved by the Ga-assisted method on native oxide-covered $\mathrm{Si}(111)$ substrates (step 1 shown in Figure 1). Vertical nanowire growth has been

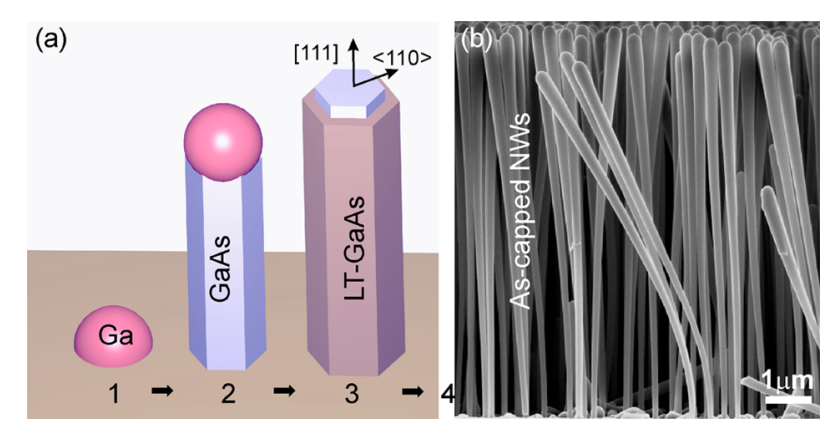

Figure 1. (a) Growth steps to obtain a GaAs/LT-GaAs core-shell nanowire structure based on the Ga-assisted vapor-liquid-solid mechanism. (1) Ga seed droplet, (2) growth of a high-quality GaAs NW, (3) followed by the consumption of the Ga droplet and the growth a of low-temperature GaAs shell. In step 3, the top of the nanowire is not shown to better highlight its core-shell structure. (b) SEM of the nanowire array after encapsulation of the wires with a thin amorphous As layer (step 4) for protection against air exposure needed to further perform atomic-scale characterization of the shell.

achieved by engineering the native oxide thickness and adjusting the growth conditions. ${ }^{20}$ Growth is started by simultaneous opening of gallium and arsenic shutters once the growth temperature is reached. The growth of the NW core was carried out at a nominal growth rate of $0.3 \AA / \mathrm{s}$, an $\mathrm{As}_{4}$ partial pressure of $2.5 \times 10^{-6} \mathrm{mbar}$, Ga partial pressure of 1.42 $\times 10^{-7} \mathrm{mbar}$, and a temperature of $640^{\circ} \mathrm{C}$ (step 2) for $90 \mathrm{~min}$. The Ga droplet was then consumed by closing the Ga shutter for $30 \mathrm{~min}$ under $\mathrm{As}_{4}$ flux. The temperature was subsequently reduced to $300{ }^{\circ} \mathrm{C}$. Once stabilized, a shell was grown at a nominal growth rate of $1.1 \AA / \mathrm{s}$ and an $\mathrm{As}_{4}$ partial pressure of $1.18 \times 10^{-5}$ mbar (step 3). At the end of the growth, the Ga flux was stopped, and the temperature was further lowered to room temperature, to cap the NWs with a thin As layer for protection against air exposure (step 4). ${ }^{21}$ For comparison, GaAs NWs were also grown using the same conditions (steps 1 and 2), but without the LT-GaAs shell, or were capped with a 4 $\mathrm{nm}$-thick AlGaAs shell grown at a reduced temperature of 465 ${ }^{\circ} \mathrm{C}$ and increased $\mathrm{As}_{4}$ partial pressure of $5 \times 10^{-5}$ mbar.
Figure $1 \mathrm{~b}$ shows a scanning electron microscopy (SEM) micrograph of the NWs at the end of growth. The As-capped NWs, with an average length of $8.6 \mu \mathrm{m}$, exhibit a cotton-bud shape with a capping thickness decreasing from $\sim 60 \mathrm{~nm}$ in the top region of the NWs to $\sim 20 \mathrm{~nm}$ in the lower part (see Figure S1 and S2e in the Supporting Information). This shape results from the geometrically limited flux of $\mathrm{As}_{4}$ into the dense NW array, which is a known effect in NW array growth called shadowing. After growth, the NWs were transferred to another ultra high vacuum system to be characterized with STM at a temperature $T$ of 77 and $300 \mathrm{~K}$. Prior to the STM measurements, the capping layer was desorbed at $350{ }^{\circ} \mathrm{C}$ for $1 \mathrm{~h}$, a temperature much lower than the congruent evaporation temperature of GaAs. ${ }^{22}$ Then, the NWs were cleaved off in situ and deposited on a thin $\mathrm{Ag}$ film grown on a $\mathrm{Si}(111)$ surface.

A flat lying nanowire is shown in the SEM image of Figure 2a. Three sidewalls are visible, consistent with the overall hexagonal shape of the NW indicated schematically at the right end. $^{23}$ On the top sidewall, small fluctuations of the bright contrast point to corrugated sidewalls. This roughness is also

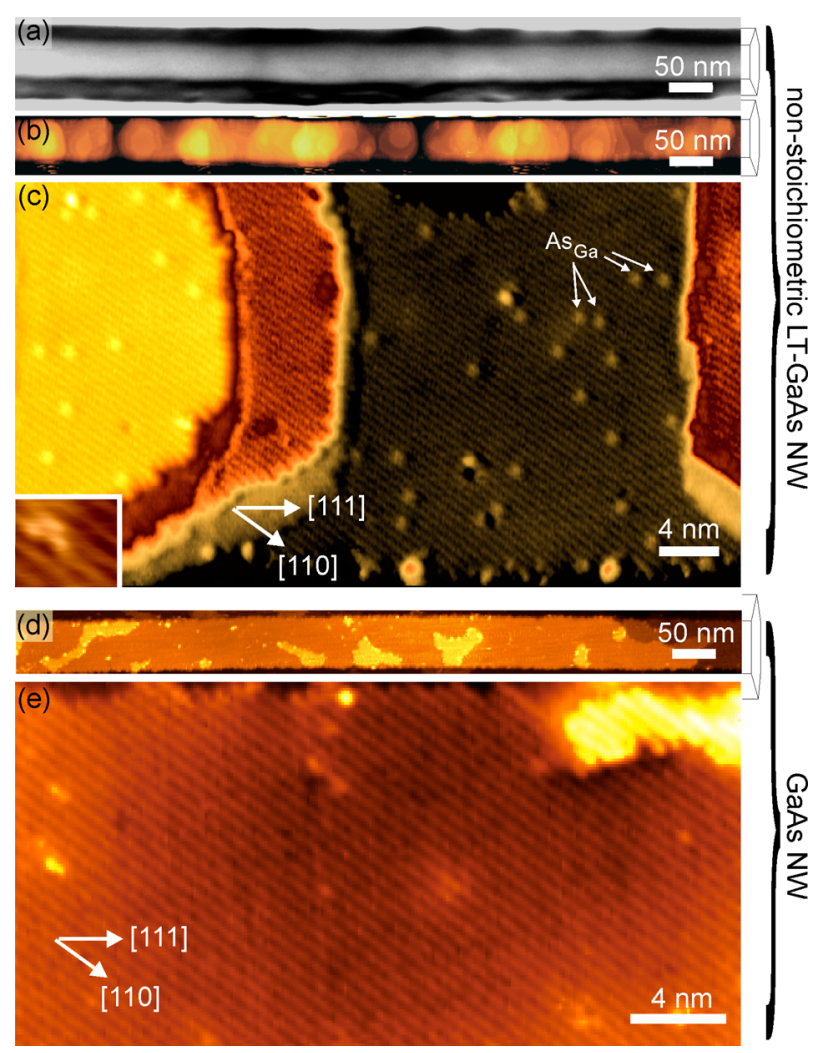

Figure 2. (a) SEM and (b) STM images of a single GaAs/LT-GaAs $\mathrm{NW}$ transferred onto a $\mathrm{Ag} / \mathrm{Si}(111)$ surface after the sublimation of the capping layer. (c) High-resolution STM image obtained on the $\{110\}$ sidewall of a LT-GaAs shell. The arrows point to subsurface $\mathrm{As}_{\mathrm{Ga}}$ arsenic antisites. Inset: High resolution image of an $\mathrm{As}_{\mathrm{Ga}}$ defect showing the typical structure consisting of a central core with two satellites. (d) STM image of a GaAs NW (without LT-GaAs shell) transferred onto a $\mathrm{Si}(111)$ surface after the sublimation of the capping layer. (e) High-resolution STM image obtained on the $\{110\}$ sidewall of a GaAs NW. No $\mathrm{As}_{\mathrm{Ga}}$ antisite defects are present. The SEM was operated at an accelerating potential of $10 \mathrm{kV}$, and secondary electrons were detected. Tunneling conditions in $\mathrm{b}-\mathrm{c}, \mathrm{d}-\mathrm{e}: V_{\text {sample }}=-3.0,-4.0$ $\mathrm{V}, I_{\text {tunnel }}=10,20 \mathrm{pA}$, respectively, $T=77 \mathrm{~K}$. The color scale in $\mathrm{b}, \mathrm{c}, \mathrm{d}$, and e are $88,23,17$, and $5 \AA$, respectively. 
visible for the adjacent sidewalls and clearly resolved in the STM image of the top sidewall (Figure $2 b$ ). It strongly differs from the flatness of the sidewalls of GaAs NWs, that are covered with sparse islands with one atomic step height only (see STM image in Figure 2d). As the pure GaAs NWs were grown with similar conditions but without a LT-GaAs shell, we attribute this morphological change to the low growth temperature and, to a lesser extent, to the radial homoepitaxial growth of GaAs on (110) sidewalls, that gives rise, in thin film growth, to very corrugated surfaces. ${ }^{24,25}$

Height profiles acquired along the growth axis on a length of $2 \mu \mathrm{m}$ give an average height roughness of $4 \mathrm{~nm}$. It is caused by pyramid like mounds which are confined by the two parallel edges of the sidewalls. The mounds consist of stacked atomically flat layers, separated mostly by single atomic steps, as visible in the high resolution STM image of Figure 2c. All of the layers show well-aligned atomic rows at an angle of $\sim 35^{\circ}$ with respect to the [111] axis of the NW. This is the signature of $\{110\}$ sidewalls of zinc-blende structure NWs (as confirmed by high resolution transmission electron microscopy, Supporting Information).

Remarkably, on every terrace, a large concentration of small bright protrusions, superimposed on the imaged As sublattice, are visible. We attribute these protrusions to point defects, which are only present in the LT-GaAs shell. Comparison with the sidewall of GaAs NWs that were similarly capped but grown without the LT-GaAs shell reveals the absence of this type of features (Figure 2e). At the atomic scale (inset of Figure 2c), these defects always exhibit a shape with a central core and two satellites. This is consistent with the features observed for subsurface arsenic antisites $\mathrm{As}_{\mathrm{Ga}}$ in bulk LT III-V compounds. ${ }^{26,27}$ The absence of subsurface antisites in the GaAs NWs without a LT-GaAs shell rules out the creation of antisites during the sublimation of the capping layer. Hence the antisites are incorporated during the growth of the shell at low temperature. Counting the number of antisites per surface layer yields a concentration $N_{\mathrm{DD}}$ of $(5 \pm 2) \times 10^{18} \mathrm{~cm}^{-3}$. Considering the growth temperature used here, the concentration is inline with LT-grown bulk materials ${ }^{26,27}$ and is consistent with a concentration below the detection limit of energy-dispersive X-ray spectroscopy (EDX) performed concomitantly with TEM analyses of the NW cross sections (Supporting Information).

In order to highlight the electronic structure of these defects in the shell, spatially resolved scanning tunneling spectroscopic measurements were performed on the sidewalls with a variation of the tip-sample separation during the voltage ramp. Such a technique ensures a large dynamic range of the conductance. ${ }^{28}$ In areas where no antisite is visible, measurements reveal the typical spectrum of a GaAs(110) NW sidewall ${ }^{29}$ (lower curve in Figure 3) with the valence band at negative voltages and the conduction band at positive voltages. Both bands are separated by a zero-conductance region that has the width of GaAs bulk band gap at $300 \mathrm{~K}$. Note, the Fermi level $E_{\mathrm{F}}$ at the surface of the sidewalls is positioned at midgap.

On the antisites, the spectrum also gives a value of the band gap comparable to that of bulk GaAs, and the band edges show a similar shape (upper curve in Figure 3). But, two additional peaks are now visible in the band gap region. We attribute their existence to the tunneling of electrons into or out of the band of midgap donor states induced by the antisites. In undoped LT-GaAs thick films, the Fermi level is not pinned inside the film ${ }^{30}$ and should be positioned above both the $0 /+$ and $+/++$

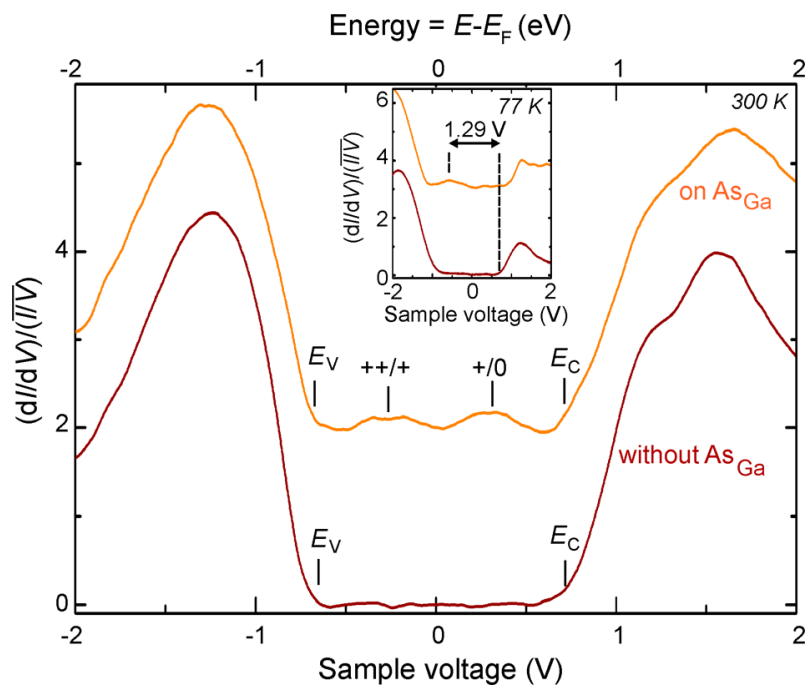

Figure 3. Tunneling spectra acquired on a subsurface antisite (upper curve) and in a region free of antisites (lower curve) at the surface of the LT-GaAs shell at a temperature of $300 \mathrm{~K}$ (inset: $77 \mathrm{~K}$ ). Tunneling conditions: $V_{\text {sample }}=-2.5 \mathrm{~V}, I_{\text {tunnel }}=10 \mathrm{pA}$. The conduction, valence band edges, and the charge transition levels are indicated by vertical dashes, labeled $E_{\mathrm{C}}$ and $E_{\mathrm{V}},++/+$, and $+/ 0$, respectively. The upper curve has been shifted for clarity.

ionization levels of the antisite, leaving the donor states completely filled, the antisite being neutral. However, the high density of steps on the $\{110\}$ sidewalls of GaAs NWs leads to a pinning of the Fermi level midgap. ${ }^{29}$ As a result, the donor states associated with subsurface antisites are partially filled to compensate the charged defect states induced by the step. The Fermi level is thus positioned midgap between both ionization levels $0 /+$ and $+/++$, and this position accounts for the occurrence of two peaks in the tunneling spectra. ${ }^{31}$ Thus, we expect that most arsenic antisites are single positively ionized in the LT-GaAs shell over a significant thickness of the shell due the strong corrugation of the sidewalls ( $\sim$ half of the shell thickness, see Figure S3 in the Supporting Information). This is in analogy with previous spectroscopic measurements on subsurface As antisites in LT grown bulk materials, ${ }^{26,27,32}$ where acceptors were used to shift the Fermi level. Thus, we can unambiguously assign the defects seen on the sidewalls of the LT-GaAs shell to subsurface arsenic antisites.

The physical impact of the antisites on the electronic and optical properties of the NWs were further investigated with pump-probe reflectivity and low-temperature cathodoluminescence (CL) experiments. The pump-probe reflectivity measurements performed at a wavelength of $820 \mathrm{~nm}$ revealed a carrier lifetime of $2.6 \mathrm{ps}$, much shorter than the typical lifetime of a few nanosecond found in GaAs/AlGaAs core-shell NWs. ${ }^{33}$ While it is consistent with the short carrier lifetime that is found for pure unpassivated GaAs $\mathrm{NWs}^{34,35}$ we have performed similar experiments for a $1.5 \mu \mathrm{m}$ thick LT-GaAs film grown on a GaAs substrate at $300{ }^{\circ} \mathrm{C}$ (see Supporting Information). The measured carrier lifetime is $1.6 \mathrm{ps}$ and results from trapping at the antisites in such thin films, ${ }^{36}$ suggesting that both surface states at the NW sidewalls and antisite defects contribute to the short lifetime in the LT-GaAs NWs.

The short lifetime due to a subsurface high recombination rate in the LT-GaAs NWs is further corroborated by the very weak signal observed in the cathodoluminescence (CL) spectrum of the As-capped LT-GaAs NWs: Figure 4 illustrates 

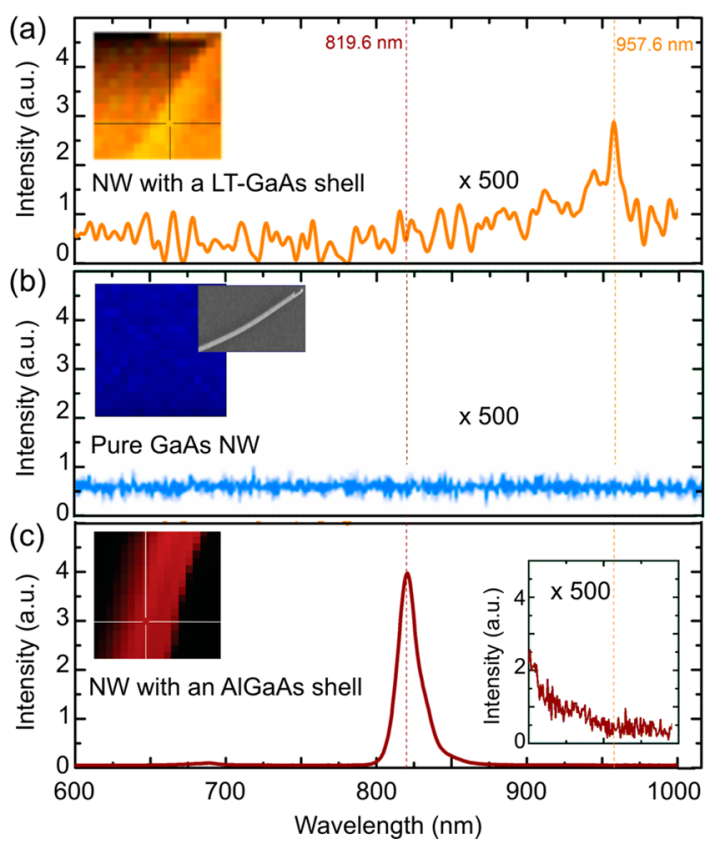

Figure 4. Low-temperature cathodoluminescence spectra of (a) a GaAs NW grown with a LT-GaAs shell, (b) a pure GaAs NW, and (c) a GaAs NW grown with an AlGaAs shell. The measurements were performed at $10 \mathrm{~K}$ with an electron beam of $10 \mathrm{kV}$ and a current of 1 nA. Inset: Cathodoluminescence mappings showing the NW location. The markers indicate the position where the spectra were acquired on the NWs. For the pure GaAs NWs that do not show any CL signal, a SEM image is also shown. The absence of the free-exiton emission at $819.6 \mathrm{~nm}$ (1.51 eV, detected only in high-quality GaAs NW with AlGaAs shell) indicates a high defect density in the LT-GaAs NW. The weak peak at $957.6 \mathrm{~nm}(1.29 \mathrm{eV})$, not seen in the inset of $(\mathrm{c})$, can be correlated to the energy separation between the $++/+$ ionization level and the conduction band measured in STS (inset Figure 3).

that the peak at $819.6 \mathrm{~nm}(1.51 \mathrm{eV})$ related to the free-exciton emission of GaAs, seen in the CL spectrum of a high-quality GaAs/AlGaAs core-shell NW, is absent in the CL spectrum of the LT-GaAs NW. Instead a new but small peak appears at $957.6 \mathrm{~nm}(1.29 \mathrm{eV})$, that is not found in pure GaAs NWs. Comparison with tunneling spectroscopic measurements performed at $77 \mathrm{~K}$ (inset of Figure 3) shows that this transition corresponds to the energy between the conduction band edge of GaAs and the +/++ ionization level, allowing to assign this sub-bandgap emission to the antisites. Hence, the LT-GaAs NW contain a high defect concentration assignable to $\mathrm{As}_{\mathrm{Ga}}$ antisites in good agreement with short carrier lifetimes and the STM results.

In addition to arsenic antisites, small clusters occur in the highest part of the NWs as visible in the SEM image of Figure 5a (inset). The filled-state STM images reveal that the clusters (protrusions in Figure 5a) are surrounded by a dark area. The observation of a dark layer around the clusters implies the existence of a depleted zone. ${ }^{37}$ This interpretation is confirmed by the tunneling spectra acquired on a well-ordered region of the sidewall and on the dark layer (spectra $i$ and ii in Figure $5 b)$. The apparent bandgap observed on the dark layer is wider, due to a smaller free carrier concentration in the depleted material. ${ }^{38}$ The clusters themselves are found to exhibit a small but steadily decrease of the conductivity in the tunneling spectrum (iii) in Figure $5 \mathrm{~b}$. This is a signature of a metallic behavior. Therefore, we attribute the dark zone surrounding the
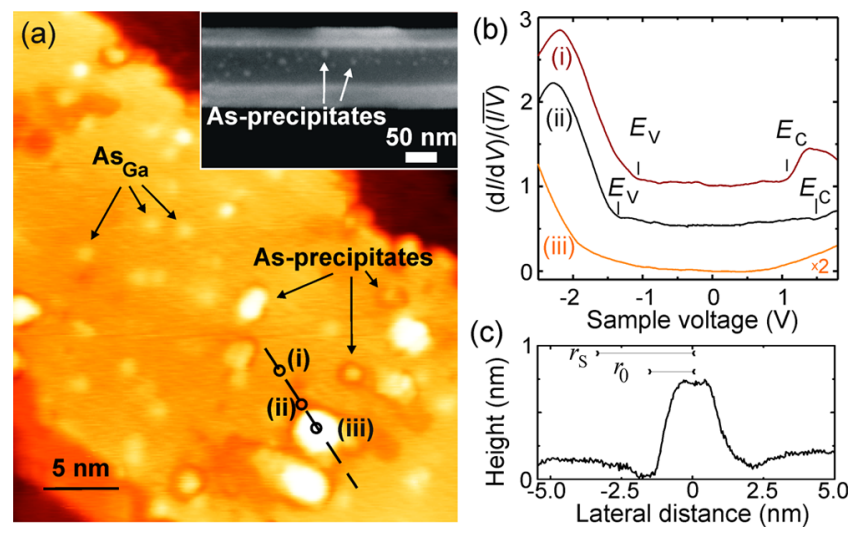

Figure 5. (a) STM and SEM (shown as inset) images of the top part of a NW transferred onto a $\mathrm{Ag} / \mathrm{Si}(111)$ surface after the sublimation of the capping layer. Several As precipitates and three $\mathrm{As}_{\mathrm{Ga}}$ arsenic antisites are highlighted. Color scale of the STM image: $15 \AA$. (b) Tunneling spectra acquired at the positions indicated in (a) by circles numbered $\mathrm{i}-\mathrm{iii}$. The conduction and valence band edges are indicated by vertical dashes and labeled $E_{\mathrm{C}}$ and $E_{\mathrm{V}}$, respectively. (c) Height profile measured along the dashed line shown in a. $r_{0}$ and $r_{s}$ correspond to the radius of the precipitate and of the positively charged sphere surrounding the precipitate. Tunneling conditions: $V_{\text {sample }}=-3.0 \mathrm{~V}, I_{\text {tunnel }}=10 \mathrm{pA}, T=77 \mathrm{~K}$.

clusters to a depletion zone arising from the formation of a Schottky barrier between a metallic cluster and the GaAs matrix, in agreement with arsenic precipitates in bulk GaAs. ${ }^{37}$ As the presence of Ga clusters can be ruled out, these clusters are identified as arsenic precipitates.

The As precipitates are able to capture electrons. The number $n$ of electrons stored in a precipitate of radius $r_{0}$ corresponds to $n=(4 \pi \varepsilon / q) r_{0} \phi_{\mathrm{b}}$, with $\varepsilon$ the permittivity of GaAs, $q$ the elementary charge, and $\phi_{\mathrm{b}}$ the built-in potential. ${ }^{39}$ In the STM image of Figure $5 \mathrm{a}$, the precipitate that is analyzed in the line profile has a radius $r_{0}$ of $1.6 \mathrm{~nm}$. Assuming a typical barrier height of $0.8 \mathrm{~V}^{39}$ and taking into account the observed midgap pinning of the Fermi level (Figure 5b), a built-in potential $\phi_{\mathrm{b}}$ of $100 \mathrm{mV}$ is deduced, corresponding to the capture of roughly one electron per precipitate. Based on the concentration of ionized arsenic antisites $N_{\mathrm{DD}}$ that appears similar in Figure 5 and Figure 2, we can then estimate the width of the depletion zone around the precipitates. The condition of charge balance implies the formation of a positively charged sphere with radius $r_{\mathrm{s}}$ around a precipitate. As a result, the negative charge $Q$ found in the precipitate is related to $r_{\mathrm{s}}$ by

$$
Q=\frac{4 \pi}{3}\left(r_{\mathrm{s}}^{3}-r_{0}^{3}\right) e N_{\mathrm{DD}}
$$

yielding $r_{\mathrm{s}}=3.5 \mathrm{~nm}$. Subtracting $r_{0}$ from $r_{\mathrm{s}}$ leads to a depleted zone, consistent with the height profile of Figure $5 \mathrm{c}$. Remarkably, when the precipitates are close enough, the depletion spheres overlap, allowing the direct visualization of the As precipitate model to account for the semi-insulating character of annealed LT-GaAs layers. ${ }^{39}$

Finally, the As precipitates were only observed on the top part of the NWs. They are known to result from the accretion of arsenic point defects upon annealing, predominantly As interstitials that are much more mobile than $\mathrm{As}_{\mathrm{Ga}}$ and can thus form precipitates at much lower temperature. ${ }^{40}$ As this incorporation of excess of arsenic is directly related to the growth temperature, ${ }^{41}$ we suspect that it is caused by an 
inhomogeneous temperature profile along the NW during the growth. Such a lower temperature at the top of the NW can be explained by heat losses that are expected to be the highest at the top part of the NW, since it is surrounded by "cold" vacuum, in contrast to the bottom that sees the hot surfaces of the surrounding NWs. Hence by a suitable choice of the growth temperature, the NW electronic properties could be tuned by point defects or precipitates leading to hopping via localized states or to a semi-insulating state, respectively.

In conclusion, we have investigated the structural, electronic, and optical properties of a LT-GaAs shell grown around GaAs NWs. Although the radial growth of the shell gives rise to rough sidewalls, the low temperature growth does not preclude the formation of a shell with a good crystallinity as deduced by the crystallographic characterization of the NW surface. It further allows the successful incorporation of excess arsenic in the GaAs matrix in the form of arsenic antisites. Depending on the annealing, the shell can also contain As precipitates. All of these defects introduce midgap states that are key elements to simultaneously obtain a short carrier lifetime and a low electrical conductivity, suggesting that the shell has the required properties for terahertz and photoconductive applications.

\section{ASSOCIATED CONTENT}

\section{S Supporting Information}

The Supporting Information is available free of charge on the ACS Publications website at DOI: 10.1021/acs.nanolett.5b01802.

TEM analysis of the nanowires, pump-probe reflectivity experiments (PDF)

\section{AUTHOR INFORMATION}

\section{Corresponding Author}

*E-mail: bruno.grandidier@isen.iemn.univ-lille1.fr.

\section{Notes}

The authors declare no competing financial interest.

\section{ACKNOWLEDGMENTS}

This study was financially supported by the European Community's Seventh Framework Program (Grant No. PITN-GA-2012-316751, "Nanoembrace" Project), the EQUIPEX program Excelsior, the RENATECH network, and the Impuls und Vernetzungsfond of the Helmholtz-Gemeinschaft Deutscher Forschungszentren (Grant No. HIRG-0014). T.X. acknowledges the support of the Region Nord-Pas-de-Calais and of the National Natural Science Foundation of China (Grant No. 61204014). T.D. acknowledges funding by DGA. F.M. and A.F.iM. thank SNF (Grant No. 143908). G.T., H.A.P. and A.F.iM. thank funding through the NCCR QSIT. A.F.iM. thanks the DAR of EPFL for funding the CL set-up. The authors thank X. Wallart for fruitful discussions.

\section{REFERENCES}

(1) Caroff, P.; Dick, K. A.; Johansson, J.; Messing, M. E.; Deppert, K.; Samuelson, L. Nat. Nanotechnol. 2009, 4, 50-55.

(2) Dheeraj, D. L.; Patriarche, G.; Zhou, H.; Hoang, T. B.; Moses, A. F.; Grønsberg, S.; Van Helvoort, A. T. J.; Fimland, B.-O.; Weman, H. Nano Lett. 2008, 8, 4459-4463.

(3) Kretinin, A. V.; Popovitz-Biro, R.; Mahalu, D.; Shtrikman, H. Nano Lett. 2010, 10, 3439-3445.

(4) Tang, J.; Huo, Z.; Brittman, S.; Gao, H.; Yang, P. Nat. Nanotechnol. 2011, 6, 568-572.

(5) Ferguson, B.; Zhang, X. C. Nat. Mater. 2002, 1, 26-33.
(6) Nagel, M.; Först, M.; Kurz, H. J. Phys.: Condens. Matter 2006, 18, S601.

(7) Liu, H. B.; Zhong, H.; Karpowicz, N.; Chen, Y.; Zhang, X.-C. Proc. IEEE 2007, 95, 1514-1527.

(8) Jepsen, P. U.; Cooke, D. G.; Koch, M. Laser Photon. Rev. 2011, 5, 124-166.

(9) Tchernycheva, M.; Harmand, J. C.; Patriarche, G.; Travers, L.; Cirlin, G. E. Nanotechnology 2006, 17, 4025.

(10) Plissard, S.; Dick, K. A.; Larrieu, G.; Godey, S.; Addad, A.; Wallart, X.; Caroff, P. Nanotechnology 2010, 21, 385602.

(11) Ahn, H.; Ku, Y.-P.; Wang, Y.-C.; Chuang, C.-H.; Gwo, S.; Pan, C. L. Appl. Phys. Lett. 2007, 91, 132108.

(12) Seletskiy, D. V.; Hasselbeck, M. P.; Cederberg, J. G.; Katzenmeyer, A.; Toimil-Molares, M. E.; Léonard, F.; Talin, A. A.; Sheik-Bahae, M. Phys. Rev. B: Condens. Matter Mater. Phys. 2011, 84, 115421.

(13) Trukhin, V. N.; Buyskikh, A. S.; Kaliteevskaya, N. A.; Bourauleuv, A. D.; Samoilov, L. L.; Samsonenko, Y. B.; Cirlin, G. E.; Kaliteevski, M. A.; Gallant, A. J. Appl. Phys. Lett. 2013, 103, 072108.

(14) Lee, W. J.; Ma, J. W.; Bae, J. M.; Jeong, K. S.; Cho, M. H.; Kang, C.; Wi, J. S. Sci. Rep. 2013, 3, 3.

(15) Arlauskas, A.; Treu, J.; Saller, K.; Beleckaite, I.; Koblmüller, G.; Krotkus, A. Nano Lett. 2014, 14, 1508-1514.

(16) Peytavit, E.; Arscott, S.; Lippens, D.; Mouret, G.; Matton, S.; Masselin, P.; Bocquet, R.; Lampin, J. F.; Desplanque, L.; Mollot, F. Appl. Phys. Lett. 2002, 81, 1174-1176.

(17) Mikulics, M.; Michael, E. A.; Schieder, R.; Stutzki, J.; Güsten, R.; Marso, M.; Van der Hart, A.; Bochem, H. P.; Lüth, H.; Kordoš, P. Appl. Phys. Lett. 2006, 88, 041118.

(18) Dufouleur, J.; Colombo, C.; Garma, T.; Ketterer, B.; Uccelli, E.; Nicotra, M.; Fontcuberta i Morral, A. Nano Lett. 2010, 10, 1734-1740.

(19) Heigoldt, M.; Arbiol, J.; Spirkoska, D.; Rebled, J. M.; ConesaBoj, S.; Abstreiter, G.; Peiro, F.; Morante, J. R.; Fontcuberta i Morral, A. J. Mater. Chem. 2009, 19, 840-848.

(20) Matteini, F.; Tütüncüoglu, G.; Potts, H.; Jabeen, F.; Fontcuberta i Morral, A. Cryst. Growth Des. 2015, 15, 3105-3109.

(21) Xu, T.; Dick, K. A.; Plissard, S.; Nguyen, T. H.; Makoudi, Y.; Berthe, M.; Nys, J.-P.; Wallart, X.; Grandidier, B.; Caroff, P. Nanotechnology 2012, 23, 095702.

(22) Zhou, Z. Y.; Zheng, C. X.; Tang, W. X.; Jesson, D. E.; Tersoff, J. Appl. Phys. Lett. 2010, 97, 121912.

(23) Heiss, M.; et al. Phys. Rev. B: Condens. Matter Mater. Phys. 2011, $83,045303$.

(24) Tejedor, P.; Šmilauer, P.; Roberts, C.; Joyce, B. A. Phys. Rev. B: Condens. Matter Mater. Phys. 1999, 59, 2341.

(25) Tejedor, P.; Šmilauer, P.; Joyce, B. A. Surf. Sci. 1999, 424, L309-L313.

(26) Feenstra, R. M.; Woodall, J. M.; Pettit, G. D. Phys. Rev. Lett. 1993, 71, 1176.

(27) Grandidier, B.; Chen, H.; Feenstra, R. M.; McInturff, D. T.; Juodawlkis, P. W.; Ralph, S. E. Appl. Phys. Lett. 1999, 74, 1439-1441.

(28) Feenstra, R. M. Phys. Rev. B: Condens. Matter Mater. Phys. 1994, 50, 4561-4570.

(29) Capiod, P.; Xu, T.; Nys, J. P.; Berthe, M.; Patriarche, G.; Lymperakis, L.; Neugebauer, J.; Caroff, P.; Dunin-Borkowski, R. E.; Ebert, Ph.; Grandidier, B. Appl. Phys. Lett. 2013, 103, 122104.

(30) Warren, A. C.; Woodall, J. M.; Kirchner, P. D.; Yin, X.; Pollak, F.; Melloch, M. R.; Otsuka, N.; Mahalingam, K. Phys. Rev. B: Condens. Matter Mater. Phys. 1992, 46, 4617.

(31) De La Broise, X.; Delerue, C.; Lannoo, M.; Grandidier, B.; Stiévenard, D. Phys. Rev. B: Condens. Matter Mater. Phys. 2000, 61, 2138.

(32) Grandidier, B.; Nys, J. P.; Delerue, C.; Stievenard, D.; Higo, Y.; Tanaka, M. Appl. Phys. Lett. 2000, 77, 4001-4003.

(33) Demichel, O.; Heiss, M.; Bleuse, J.; Mariette, H.; Fontcuberta I Morral, A. Appl. Phys. Lett. 2010, 97, 201907.

(34) Parkinson, P.; Lloyd-Hughes, J.; Gao, Q.; Tan, H. H.; Jagadish, C.; Johnston, M. B.; Herz, L. M. Nano Lett. 2007, 7, 2162-2165. 
(35) Chang, C. C.; Chi, C. Y.; Yao, M.; Huang, N.; Chen, C. C.; Theiss, J.; Bushmaker, A. W.; LaLumondiere, S.; Yeh, T.-W.; Povinelli, M. L.; Zhou, C.; Dapkus, P. D.; Cronin, S. B. Nano Lett. 2012, 12, 4484-4489.

(36) Adomavičius, R.; Krotkus, A.; Bertulis, V.; Sirutkaitis, V.; Butkus, R.; Piskarskas, A. Appl. Phys. Lett. 2003, 83, 5304-5306.

(37) Feenstra, R. M.; Vaterlaus, A.; Woodall, J. M.; Pettit, G. D. Appl. Phys. Lett. 1993, 63, 2528-2530.

(38) Jäger, N. D.; Ebert, P.; Urban, K.; Krause-Rehberg, R.; Weber, E. R. Phys. Rev. B: Condens. Matter Mater. Phys. 2002, 65, 195318.

(39) Warren, A. C.; Woodall, J. M.; Freeouf, J. L.; Grischkowsky, D.; McInturff, D. T.; Melloch, M. R.; Otsuka, N. Appl. Phys. Lett. 1990, 57, 1331-1333.

(40) Melloch, M. R.; Otsuka, N.; Mahalingam, K.; Chang, C. L.; Woodall, J. M.; Pettit, G. D.; Kirchner, P. D.; Cardone, F.; Warren, A. C.; Nolte, D. D. J. Appl. Phys. 1992, 72, 3509-3513.

(41) Luysberg, M.; Sohn, H.; Prasad, A.; Specht, P.; Liliental-Weber, Z.; Weber, E. R.; Gebauer, J.; Krause-Rehberg, R. J. Appl. Phys. 1998, $83,561-566$. 